\title{
Consumo de medicamentos por trabalhadores de hospital
}

\author{
Use of medication among hospital workers
}

Tatiana Chama Borges Luz ${ }^{1}$

Vera Lucia Luiza ${ }^{2}$

Fernando Genovez Avelar ${ }^{2}$

Yara Hahr Marques Hökerberg ${ }^{3}$

Sonia Regina Lambert Passos ${ }^{3}$

${ }^{1}$ Laboratório de

Epidemiologia e

Antropologia Médica,

Centro de Pesquisa René

Rachou, Fundação Oswaldo

Cruz (LEAM/CpqRR/

Fiocruz). Av. Augusto de

Lima 1715/609, Barro

Preto. 30190-002 Belo

Horizonte MG.

tatianachama@cpqrr.fiocruz.br

${ }^{2}$ Núcleo de Assistência

Farmacêutica, Escola

Nacional de Saúde Pública

Sérgio Arouca, Fundação

Oswaldo Cruz (NAF/ENSP/

Fiocruz)

${ }^{3}$ Laboratório de

Epidemiologia Clínica,

Instituto de Pesquisa

Clínica Evandro Chagas,

Fundação Oswaldo Cruz

(IPEC/Fiocruz)

\begin{abstract}
Although medication is acknowledged as a key element in treating health problems, there is little information available on the use of medication by hospital workers. To estimate the prevalence and describe the patterns of medication consumption by hospital workers and to identify the factors associated with such consumption in this population, data from the "PROSEC" baseline cohort were analyzed $(n=417)$. The prevalence of overall medication consumption was $72.4 \%$, most of which was for nervous complaints (25.4\%), especially analgesics (17.8\%). Use of any amount of medication was independently associated with gender, number of medically diagnosed conditions and health problem in the two weeks prior to the interview. Use of a drug was significantly associated with income whereas self-diagnosed health problems were independently related with the use of two or more pharmaceutical products. The high prevalence of medication usage in this population, with analgesics being the most consumed medication, should be seen as a cause for concern, since many consumers are unaware that these products are not exempt from risk. Women and individuals in poor health are the main candidates for intervention programs in order to promote adequate and proper use of these pharmaceutical products.
\end{abstract}

Key words Use of medication, Associated factors, Workers
Resumo Medicamentos são um componente essencial da terapêutica, no entanto, informações sobre seu consumo em população trabalhadora são escassas. Para estimar a prevalência, caracterizar o perfil daqueles consumidos e avaliar os fatores associados ao seu uso em trabalhadores foi realizado estudo seccional de dados da linha de base da Coorte "PROSEC" $(n=417)$. A prevalência de consumo de pelo menos um medicamento foi de 72,4\%, destacando-se fármacos com ação no sistema nervoso $(25,4 \%)$, especialmente analgésicos (17,8\%). Fatores associados ao uso de qualquer número de medicamentos $(p<0,05)$ foram sexo, número de condições diagnosticadas por médico e problema de saúde nos quinze dias anteriores à entrevista. A renda esteve significativamente associada ao consumo de um medicamento ao passo que autoavaliação de saúde apresentou associação independente com o consumo de dois ou mais produtos. A alta prevalência de uso de medicamentos nesta população, particularmente de analgésicos, requer atenção, pois muitos usuários costumam desconhecer ou relegar o fato de que tais fármacos não são isentos de risco. Mulheres e indivíduos em pior estado de saúde constituem os principais candidatos a programas de intervenção visando o uso adequado de produtos farmacêuticos.

Palavras-chave Uso de medicamentos, Fatores associados, Trabalhadores 


\section{Introdução}

Os avanços nas pesquisas e produção de medicamentos ocorridos especialmente após a segunda guerra mundial, acompanhados de extensiva promoção comercial desses produtos tornaram a indústria farmacêutica um dos segmentos mais lucrativos da atualidade. Somente no ano de 2008, o mercado farmacêutico mundial movimentou cifras da ordem de 773 bilhões $^{1}$. No Brasil, esse mercado fechou o ano de 2009 com 33,6 bilhões de reais das vendas nominais, correspondendo a US\$ 17,1 bilhões, com 1,8 bilhões de unidades vendidas ${ }^{2}$.

É fato que os medicamentos são um componente essencial da terapêutica e que desempenham um reconhecido papel na recuperação e na manutenção da saúde e no bem-estar geral dos usuários desses produtos. Por outro lado, a crença excessiva em seu poder terapêutico ${ }^{3,4}$ os levaram à condição de mercadoria especial, lastreada pelo conhecimento científico como único modo válido de se obter saúde ${ }^{4,5}$.

Os tratamentos medicamentosos não são invariavelmente seguidos de melhora clínica e vários problemas têm sido relacionados a este consumo, tais como: automedicação; prescrição e/ou utilização sem indicação terapêutica, com posologia e duração do tratamento divergentes do preconizado; reações adversas e intoxicações; além do aumento da procura por serviços de urgência e emergência que pode gerar hospitalizações e até mesmo a mortes ${ }^{6,7}$.

Existem ainda, diferenças quanto ao padrão de consumo de medicamentos na sociedade. Alguns estudos têm mostrado que a utilização destes produtos é maior para mulheres ${ }^{8-11}$, idosos ${ }^{8,9}$, indivíduos com maior escolaridade ${ }^{12}$ e renda $^{8}$, indivíduos com plano de saúde suplementar ${ }^{13}$, com pior autoavaliação do estado de saúde ${ }^{8-10}$, com presença de doenças ${ }^{8-10}$, entre outros fatores ${ }^{14}$.

Desse modo, a população trabalhadora é um grupo populacional que merece atenção especial, pois estudos realizados em diversas partes do mundo mostraram que algumas características do processo de trabalho, como responsabilidade excessiva, ocorrência de situações estressantes, falta de segurança e de estabilidade no emprego, carga horária intensa, entre outras, estão associadas a um pior estado de saúde e à ocorrência de diversas doenças e agravos à saúde ${ }^{15-17}$.

A literatura evidencia ainda que os profissionais que atuam em hospitais relatam estresse e insatisfação profissional ${ }^{18-19}$, os quais têm sido associados a sintomas como fadiga, desânimo, ansiedade e irritação ${ }^{20-22}$. Há queixas também com relação ao conforto oferecido aos profissionais, qualidade dos equipamentos, materiais de trabalho e salário ${ }^{18}$.

Diante desse contexto, é plausível supor que os medicamentos sejam um recurso terapêutico bastante utilizado pela população trabalhadora, mas ainda são escassas na literatura informações sobre este tema ${ }^{11}$ e alguns estudos permanecem restritos à avaliação do uso, entre trabalhadores, de algumas classes de medicamentos específicas, tais como psicotrópicos, benzodiazepínicos e anti-inflamatórios não esteroidais ${ }^{23-25}$.

No Brasil, apesar da relevância de pesquisas epidemiológicas sobre uso de medicamentos, poucos são os estudos disponíveis ${ }^{26}$, e ainda são raros os estudos que apresentam a população trabalhadora como população-alvo ${ }^{24,27,28}$.

Assim, baseado neste painel, a presente investigação é parte da linha de base de uma coorte de trabalhadores em hospital no Estado do Rio de Janeiro, Brasil (Programa PROSEC) e referese ao estudo da prevalência e dos fatores associados ao consumo de medicamentos entre os trabalhadores desta unidade. O estudo analisa o padrão de consumo de medicamentos pelos trabalhadores, com os objetivos de: (1) estimar sua prevalência e seu consumo total; (2) identificar os mais consumidos; e (3) investigar as características sociodemográficas, as condições de saúde, bem como as condições de trabalho associadas ao seu uso.

\section{Metodologia}

\section{Delineamento, coleta de dados e população do estudo}

O presente estudo analisou de forma seccional os dados da linha de base do Estudo de Coorte em Trabalhadores do Instituto de Pesquisa Clínica Evandro Chagas - Programa de Saúde do IPEC (PROSEC), obtidos entre 2004 e 2005, por meio de questionário estruturado autopreenchível, aplicado no local de trabalho por equipe previamente treinada. Este estudo foi aprovado pelo Comitê de Ética do IPEC.

A população-alvo foi constituída de todos os trabalhadores nesta instituição de saúde e pesquisa em doenças infecciosas. Excluíram-se os trabalhadores inativos, os com permanência prevista menor do que dois anos e os cedidos a outras instituições. De uma população estimada de 497 trabalhadores em efetivo exercício, 424 
A variável dependente do estudo foi o número de medicamentos consumidos nas duas últimas semanas. Foram investigados o nome do medicamento, a concentração e a forma farmacêutica. Solicitou-se, ainda, ao entrevistado, que utilizasse sua receita, embalagem e/ou bulas dos produtos para o preenchimento do questionário. Estagiários de farmácia treinados foram apresentados ao participante para auxiliar no preenchimento destas informações, apenas se fossem solicitados pelo mesmo, durante o autopreenchimento do questionário. Tinham como suporte um manual de procedimentos padronizado para este estudo e um supervisor de campo.

Os medicamentos foram posteriormente identificados empregando-se o Dicionário de Especialidades Farmacêuticas DEF 2004/2005 29 e o banco de dados da Agência Nacional de Vigilância Sanitária (ANVISA) ${ }^{30}$. O sistema de codificação anatômico-terapêutico-químico (ATC) do World Health Organization Collaborating Centre for Drugs Statistics Methodology (WHO CC DSM) $)^{31}$ foi utilizado para classificar os medicamentos, levando-se em consideração três informações: os nomes dos princípios ativos, a dose e a forma farmacêutica.

\section{Variáveis independentes}

Três conjuntos de variáveis foram considerados: (1) sociodemográficas e econômicas: sexo, idade, escolaridade, cor da pele autorreferida, estado conjugal, renda pessoal mensal e cobertura por medicina suplementar; (2) indicadores da condição de saúde: autoavaliação da saúde, número de doenças / condições diagnosticadas por médico, índice de massa corporal (IMC), ocorrência de problema de saúde nas duas últimas semanas, interrupção das atividades habituais por problemas de saúde nas duas últimas semanas, internações hospitalares nos últimos 12 meses. A autoavaliação da saúde foi medida por meio da seguinte pergunta: De um modo geral, em comparação a pessoas da sua idade, como você considera o seu próprio estado de saúde com as seguintes opções de resposta (1) muito bom (2) bom (3) regular (4) ruim. Ressalta-se que mes- mo sendo medida por meio de uma única pergunta, há evidências de que a autoavaliação de saúde alcance várias dimensões desta e que um processo de ponderação destas dimensões é desenvolvido pelos indivíduos ao respondê- $\mathrm{la}^{32}$. Este indicador tem sido amplamente utilizado em inquéritos populacionais por ser de fácil aplicação, permitir comparações internacionais e ainda por sua capacidade preditiva tanto da morbidade quanto da mortalidade ${ }^{32-34}$.

O número de doenças / condições foi baseado no relato de diagnóstico médico prévio de hipercolesterolemia, diabetes mellitus, infarto do miocárdio, angina pectoris, acidente vascular cerebral, asma, bronquite, cálculo renal, cálculo vesical, úlcera estomacal ou duodenal, gastrite, lesão por esforço repetitivo, artrite ou reumatismo, hérnia de disco, hipertireoidismo, hipertensão arterial e tuberculose; (3) indicadores relacionados ao trabalho: ocupação atual, trabalho em turnos, carga horária trabalhada na semana, exposição à agente químico, físico, biológico, ergonômico ou psicossocial no ambiente de trabalho, licença por motivo de saúde relacionada ao trabalho nos últimos 12 meses. A ocupação atual foi autorreferida pelos respondentes e posteriormente codificada segundo a Classificação Brasileira de Ocupações (CBO 2002) ${ }^{35}$.

\section{Análise estatística}

A análise estatística foi baseada no teste do qui-quadrado de Pearson para comparação de proporções e em razões de chance estimadas pela regressão logística multinomial ${ }^{36}$. O número de medicamentos consumidos foi categorizado em três níveis: nenhum; um; e dois ou mais medicamentos, sendo a ausência de consumo a categoria de referência. Para a análise multivariada, foram incluídas no modelo inicial todas as variáveis que na análise bivariada apresentaram associação com o uso de medicamentos em nível de significância inferior a 0,20, de acordo com o teste do qui-quadrado de Pearson. Permaneceram no modelo final aquelas variáveis associadas à dependente em nível de significância inferior a 0,05. A análise estatística foi realizada utilizandose o programa Stata, versão 10.0 (Stata Corporation, College Station, Estados Unidos).

\section{Resultados}

A prevalência de consumo de pelo menos um medicamento foi de $72,4 \%$, variando a quanti- 
dade consumida entre um e seis. A maioria $(54,1 \%)$ relatou ter feito uso de apenas um produto, $30,5 \%$ reportaram o uso de dois, $12,9 \%$ de três e os demais utilizaram entre 4 e 6.

Foram identificadas 460 diferentes especialidades farmacêuticas citadas pelos respondentes. Dentre os medicamentos mais consumidos destacaram-se aqueles com ação no sistema nervoso $(25,4 \%)$, sobretudo analgésicos, seguidos por produtos com ação no sistema cardiovascular $(18,3 \%)$, representados principalmente pelos agentes que atuam sobre o sistema renina-angi- otensina. Os medicamentos para o trato alimentar e o metabolismo e aqueles com ação no sistema genitourinário e hormônios sexuais apareceram em terceiro $(16,1 \%)$ e quarto lugares $(14,8 \%)$, respectivamente (Tabela 1$)$.

Nas Tabelas 2, 3 e 4 estão apresentados os resultados da análise bivariada da associação entre o número de medicamentos consumidos e as covariáveis consideradas neste estudo. Associações diretas $(\mathrm{p}<0,05)$ foram observadas com sexo feminino, idade $>42$ anos e acesso à cobertura por medicina suplementar (Tabela 2).

Tabela 1. Medicamentos utilizados segundo a classificação anátomo-terapêutica (níveis 1 e 2 do Sistema de Classificação ATC), hospital terciário, Rio de Janeiro/RJ, 2004.

\begin{tabular}{|c|c|c|c|}
\hline Medicamentos & Código ATC & $\mathbf{N}$ & $\%$ \\
\hline Sistema Nervoso & $\mathbf{N}$ & 117 & 25,4 \\
\hline Analgésicos & N02 & 82 & 17,8 \\
\hline Psicoanalépticos & N06 & 14 & 3,0 \\
\hline Psicolépticos & N05 & 12 & 2,6 \\
\hline Antiepilépticos & N03 & 7 & 1,5 \\
\hline Outros medicamentos com ação no sistema nervoso & N04 - N07 & 2 & 0,4 \\
\hline Sistema cardiovascular & $\mathrm{C}$ & 84 & 18,3 \\
\hline Agentes sobre o sistema renina-angiotensina & $\mathrm{C} 09$ & 27 & 5,9 \\
\hline Betabloqueadores & $\mathrm{C} 07$ & 17 & 3,7 \\
\hline Diuréticos & $\mathrm{C} 03$ & 14 & 3,0 \\
\hline Antilipêmicos & $\mathrm{C} 10$ & 11 & 2,4 \\
\hline Bloqueadores de canal de cálcio & $\mathrm{C} 08$ & 9 & 2,0 \\
\hline Outros & $\mathrm{C} 05-\mathrm{C} 02-\mathrm{C} 01$ & 6 & 1,3 \\
\hline Trato alimentar e metabolismo & A & 74 & 16,1 \\
\hline Vitaminas & A 11 & 35 & 7,6 \\
\hline Medicamentos para desordens gastrointestinais & A 03 & 8 & 1,7 \\
\hline Antiácidos/antiulcerosos/antiflatulentos & $\mathrm{A} 02$ & 7 & 1,5 \\
\hline Suplementos minerais & A 12 & 7 & 1,5 \\
\hline Antiobesidade, exceto produtos dietéticos & A08 & 6 & 1,3 \\
\hline Antidiabéticos & $\mathrm{A} 10$ & 5 & 1,1 \\
\hline Outros & A04 - A06-A07-A16 & 6 & 1,3 \\
\hline Sistema genitourinário e hormônios sexuais & G & 68 & 14,8 \\
\hline Hormônios sexuais e moduladores do sistema genital & G03 & 67 & 14,6 \\
\hline Antiinfecciosos e antisépticos ginecológicos & G01 & 1 & 0,2 \\
\hline Sistema músculo-esquelético & $\mathbf{M}$ & 31 & 6,7 \\
\hline Antiinflamatórios e antireumáticos & M01 & 25 & 5,4 \\
\hline Outros & M03-M04-M05 & 6 & 1,3 \\
\hline Antiinfecciosos de uso sistêmico & J & 19 & 4,1 \\
\hline Antibacterianos de uso sistêmico & $\mathrm{J} 01$ & 18 & 3,9 \\
\hline Antivirais de uso sistêmico & $\mathrm{J} 05$ & 1 & 0,2 \\
\hline Sangue e sistema hematopoiético & B & 18 & 3,9 \\
\hline Antianêmicos & B03 & 12 & 2,6 \\
\hline Antitrombóticos & B01 & 6 & 1,3 \\
\hline Sistema respiratório & $\mathbf{R}$ & 18 & 3,9 \\
\hline Antihistamínicos de uso sistêmico & R06 & 11 & 2,4 \\
\hline Outros & R01-R03-R05 & 7 & 1,5 \\
\hline Outros medicamentos & H-D-S-P-L & 31 & 6,7 \\
\hline Total & & 460 & 100,0 \\
\hline
\end{tabular}


Tabela 2. Análise bivariada da associação entre o uso de medicamentos e características sociodemográficas e econômicas. Hospital terciário, Rio de Janeiro, RJ, 2004.

\begin{tabular}{|c|c|c|c|}
\hline Variável & $\begin{array}{c}\text { Usou } 1 \\
\text { medicamento } \\
\text { OR (IC95\%) }\end{array}$ & $\begin{array}{l}\text { Usou 2ou mais } \\
\text { medicamentos } \\
\text { OR (IC } 95 \%)\end{array}$ & Valor de $\mathrm{p}^{*}$ \\
\hline \multicolumn{4}{|l|}{ Sexo } \\
\hline Masculino & 1,00 & 1,0 & 0,000 \\
\hline Feminino & $2,23(1,34-3,73)$ & $03,38(1,91-5,95)$ & \\
\hline \multicolumn{4}{|l|}{ Idade } \\
\hline $19-26$ & 1,00 & 1,00 & 0,002 \\
\hline $27-35$ & $1,32(0,68-2,57)$ & $1,54(0,74-3,21)$ & \\
\hline $36-45$ & $0,79(0,41-1,50)$ & $1,02(0,50-2,01)$ & \\
\hline $42 \mathrm{e}+$ & $2,50(1,15-5,42)$ & $4,68(2,11-10,41)$ & \\
\hline \multicolumn{4}{|l|}{ Escolaridade } \\
\hline Até ensino médio & 1,00 & 1,00 & 0,083 \\
\hline Superior & $1,62(0,91-2,88)$ & $1,80(0,95-3,42)$ & \\
\hline Pós-graduação & $1,03(0,55-1,52)$ & $2,52(1,33-4,79)$ & \\
\hline \multicolumn{4}{|l|}{ Cor da pele } \\
\hline Branca & 1,00 & 1,00 & 0,299 \\
\hline Não Branca & $0,83(0,51-1,36)$ & $0,68(0,41-1,14)$ & \\
\hline \multicolumn{4}{|l|}{ Estado conjugal } \\
\hline Casado & 1,00 & 1,00 & 0,451 \\
\hline Não casado & $1,23(0,75-2,01)$ & $0,94(0,56-1,56)$ & \\
\hline \multicolumn{4}{|c|}{ Renda pessoal mensal (salários mínimos) ${ }^{* *}$} \\
\hline$\leq 6$ & 1,00 & 1,00 & 0,027 \\
\hline Entre 6 e 12 & $2,29(1,25-4,18)$ & $1,37(0,70-2,68)$ & \\
\hline$>12$ & $1,52(0,83-2,77)$ & $2,32(1,28-4,22)$ & \\
\hline \multicolumn{4}{|c|}{ Cobertura por medicina suplementar } \\
\hline Sim & 1,00 & 1,00 & 0,005 \\
\hline Não & $0,59(0,35-0,98)$ & $(0,23-0,71)$ & \\
\hline
\end{tabular}

"Teste do qui-quadrado de Pearson * Salário mínimo nacional vigente à época da pesquisa (1 SM = R\$260,00)

Uma pior autoavaliação da saúde, a referência a pelo menos uma doença ou a condição previamente diagnosticada por médico e a apresentação de problema de saúde nas duas semanas anteriores ao preenchimento do questionário estavam significativamente associados ao uso de medicamentos $(\mathrm{p}<0,05)$ (Tabela 3$)$.

Com referência aos indicadores relacionados ao trabalho apenas a licença por motivo de saúde nos últimos 12 meses esteve diretamente associada ao uso de medicamentos $(\mathrm{p}<0,05)$ (Tabela 4).

Os resultados finais da análise multinomial dos fatores associados ao consumo de medicamentos estão apresentados na Tabela 5. Sexo (feminino), número de condições previamente diagnosticadas por médico (duas ou mais) e apresentação de problema de saúde nas duas semanas anteriores ao preenchimento do questionário apresentaram associações positivas e independentes com o consumo de medicamentos nos dois níveis considerados. Associação significativa com o consumo de 1 medicamento foi encontrada para renda entre 6 e 12 salários mínimos. Já para o consumo de 2 ou mais medicamentos foram encontradas associações significantes para pior percepção da saúde (razoável e ruim/muito ruim) e para a presença de pelo menos uma condição previamente diagnosticada por médico.

\section{Discussão}

O consumo de medicamentos nesta população de trabalhadores foi superior à prevalência relatada em estudos nacionais com população adul$\operatorname{ta}^{9,13}$ e de trabalhadores ${ }^{27}$, bem como em estudos internacionais com população adulta ${ }^{37}$, geral ${ }^{10} \mathrm{e}$ trabalhadora $^{11}$, a qual variou entre $30 \%$ e $65 \%$. Estes estudos guardam entre si algumas diferenças metodológicas que dificultam a comparação 
Tabela 3. Análise bivariada da associação entre o uso de medicamentos e indicadores da condição de saúde e do uso de serviços de saúde. Hospital terciário, Rio de Janeiro, RJ, 2004.

\begin{tabular}{|c|c|c|c|}
\hline Variável & $\begin{array}{l}\text { Usou } 1 \\
\text { medicamento } \\
\text { OR (IC95\%) }\end{array}$ & $\begin{array}{l}\text { Usou 2ou mais } \\
\text { medicamentos } \\
\text { OR (IC95\%) }\end{array}$ & Valor de $\mathbf{p}^{*}$ \\
\hline \multicolumn{4}{|l|}{ Autoavaliação } \\
\hline Muito boa & 1,00 & 1,00 & 0,000 \\
\hline Boa & $1,90(1,13-3,21)$ & $3,8(2,05-7,12)$ & \\
\hline Ruim/Muito ruim & $3,6(1,40-9,24)$ & $12,3(4,7-32,4)$ & \\
\hline \multicolumn{4}{|c|}{$\begin{array}{l}\text { Número de condições diagnosticadas por } \\
\text { médico }\end{array}$} \\
\hline Nenhuma & 1,00 & 1,00 & 0,000 \\
\hline 1 & $1,60(0,90-2,86)$ & $2,93(1,46-5,89)$ & \\
\hline $2 \mathrm{ou}+$ & $3,56(1,87-6,77)$ & $12,26(5,96-25,2)$ & \\
\hline \multicolumn{4}{|l|}{$\operatorname{IMC}\left(\mathrm{Kg} / \mathrm{m}^{2}\right)$} \\
\hline Normal (até 25) & 1,00 & 1,00 & 0,159 \\
\hline Sobrepeso $(25$ a 29,9$)$ & $1,32(0,74-2,38)$ & $1,26(0,68-2,32)$ & \\
\hline Obesidade (maior que 30) & $1,57(0,66-3,76)$ & $2,58(1,11-5,98)$ & \\
\hline \multicolumn{4}{|c|}{$\begin{array}{l}\text { Apresentou problema de saúde nas duas } \\
\text { últimas semanas }\end{array}$} \\
\hline Não & 1,00 & 1,00 & 0,000 \\
\hline Sim & $3,16(1,68-5,92)$ & $6,19(3,28-11,67)$ & \\
\hline \multicolumn{4}{|c|}{$\begin{array}{l}\text { Ficou impedido de realizar alguma de suas } \\
\text { atividades habituais (trabalho, estudo ou } \\
\text { lazer) nas duas últimas semanas }\end{array}$} \\
\hline Não & 1,00 & 1,00 & 0,226 \\
\hline Sim & $1,48(0,72-3,06)$ & $1,65(0,78-3,46)$ & \\
\hline
\end{tabular}

"Teste do qui-quadrado de Pearson

direta dos resultados, tais como a distribuição por sexo e idade da população estudada, além da forma de avaliação do uso de medicamentos. Por exemplo, Boeuf-Cazou et al. ${ }^{11}$ avaliaram um período recordatório de uma semana para o uso de medicamentos, enquanto Carvalho et al. ${ }^{9}$ somente classificaram como usuários, os indivíduos que possuíam as embalagens dos medicamentos citados.

Mesmo frente a estas limitações, nota-se que a prevalência do consumo de medicamentos na população estudada foi bastante elevada, semelhante, por exemplo, ao valor estimado por Loyola Filho et al..$^{38}$ para a de idosos $(72,1 \%)$, o que é preocupante, uma vez que a deste estudo é trabalhadora, considerada mais saudável quando comparada a outras que não trabalham, uma vez que a possibilidade do "viés de trabalhador saudável" deve ser considerada em estudos epidemiológicos conduzidos entre pessoas empregadas ${ }^{39}$.

A classe de medicamentos mais utilizada em nosso estudo, substâncias com ação no sistema nervoso pela classificação ATC, especialmente analgésicos, coincide com a identificada em outros estudos ${ }^{9-11,27,40,41}$. Entretanto, entre estes, apenas Boeuf-Cazou et al. ${ }^{11}$ classificaram os medicamentos segundo a ATC, que é proposta pela OMS para, entre outras coisas, facilitar comparações internacionais ${ }^{42}$. Ressalta-se que o manejo da dor pode ser alcançado através de medidas não farmacológicas. Os analgésicos, ainda que geralmente bem tolerados, são medicamentos apenas sintomáticos e não isentos de risco, podendo mascarar doenças subjacentes, exacerbar sintomas dolorosos, gerar enxaqueca crônica ${ }^{43} \mathrm{e}$ causar danos gástricos ${ }^{44}$, hepáticos ${ }^{45,46}$ e renais ${ }^{46}$.

Neste estudo, as características sociodemográficas e econômicas, e os indicadores da condição de saúde, contribuíram para explicar o uso de medicamentos. O sexo feminino se manteve independentemente associado ao seu consumo, resultado consistente com o observado por diversos autores $^{8,10,11}$. Razões de ordem fisiológica inerentes a este sexo, como a menstruação, a contracepção e a gravidez podem estar na origem das diferenças observadas quanto explicar o 
Tabela 4. Análise bivariada da associação entre o uso de medicamentos e indicadores relativos ao trabalho. Hospital terciário, Rio de Janeiro, RJ, 2004.

\begin{tabular}{|c|c|c|c|}
\hline Variável & $\begin{array}{c}\text { Usou } 1 \\
\text { medicamento } \\
\text { OR (IC95\%) }\end{array}$ & $\begin{array}{l}\text { Usou 2ou mais } \\
\text { medicamentos } \\
\text { OR (IC95\%) }\end{array}$ & Valor de $\mathbf{p}^{*}$ \\
\hline \multicolumn{4}{|l|}{ Ocupação no IPEC } \\
\hline Profissional de nível superior e gestão & 1,00 & 1,00 & 0,277 \\
\hline Profissional de serviços administrativos & $0,74(0,36-1,54)$ & $0,52(0,25-1,11)$ & \\
\hline Profissional de nível médio & $1,04(0,55-2,00)$ & $0,61(0,31-1,21)$ & \\
\hline Serviços Gerais & $0,53(0,25-1,12)$ & $0,45(0,21-0,96)$ & \\
\hline Outros $^{* *}$ & $1,25(0,42-3,67)$ & $0,71(0,22-2,29)$ & \\
\hline \multicolumn{4}{|l|}{$\begin{array}{l}\text { Atualmente trabalha em algum plantão } \\
\text { noturno ou de } 24 \text { horas }\end{array}$} \\
\hline Não & 1,00 & 1,00 & 0,976 \\
\hline Sim & $0,74(0,33-1,67)$ & $1,10(0,50-2,39)$ & \\
\hline \multicolumn{4}{|l|}{ Horas trabalhadas por semana } \\
\hline Até 20 & 1,00 & 1,00 & 0,629 \\
\hline 21 a 40 & $0,71(0,32-1,59)$ & $0,90(0,38-2,17)$ & \\
\hline 41 a 60 & $0,60(0,25-1,44)$ & $0,82(0,32-2,10)$ & \\
\hline 61 a 80 & $0,92(0,18-4,58)$ & $2,26(0,48-10,64)$ & \\
\hline \multicolumn{4}{|l|}{$\begin{array}{l}\text { Exposição a algum agente no ambiente } \\
\text { de trabalho }\end{array}$} \\
\hline Não & 1,00 & 1,00 & 0,968 \\
\hline Sim & $0,87(0,45-1,71)$ & $1,14(0,55-2,35)$ & \\
\hline \multicolumn{4}{|l|}{$\begin{array}{l}\text { Licença por motivo de saúde relacionada } \\
\text { ao trabalho nos últimos } 12 \text { meses }\end{array}$} \\
\hline Não & 1,00 & 1,00 & 0,015 \\
\hline Sim & $2,32(0,88-6,08)$ & $4,19(1,65-10,68)$ & \\
\hline
\end{tabular}

* Teste do qui-quadrado de Pearson ** estudantes e estagiários ${ }^{* * *}$ agente químico, físico, biológico, ergonômico ou psicossocial

maior ao consumo de medicamentos neste subgrupo. Além disso, as mulheres procuram mais os serviços de saúde do que os homens, o que aumenta as chances de receberem prescrições médicas ${ }^{47}$. Elas são, ainda, as principais responsáveis pelos cuidados com a saúde na família, o que, por conseguinte, as torna mais familiarizadas com os produtos farmacêuticos ${ }^{48}$.

A associação entre a renda e o uso de medicamentos, verificada em nosso estudo, também está de acordo com a literatura, ainda que restrita à categoria intermediária de renda. Sabe-se que os altos custos dos medicamentos constituem um dos principais moduladores de seu uso, especialmente em países em desenvolvimento ${ }^{49}$ e em países desenvolvidos cujos sistemas de financiamento deles não oferecem cobertura para toda população ${ }^{50,51}$. No Brasil, por exemplo, diversos estudos evidenciam a importância da melhor situação econômico-financeira para o maior consumo de produtos farmacêuticos ${ }^{8,9,13}$. O impacto gerado pelos gastos com medicamentos é bastante relevante. Um estudo realizado com dados da Pesquisa de Orçamentos Familiares (POF) e da Pesquisa Nacional por Amostra de Domicílios (PNAD) revelou que tais gastos constituem a maior fonte de despesas que as famílias brasileiras realizam com saúde ${ }^{52}$. Além disso, estudos que avaliam a disponibilidade de medicamentos nas farmácias públicas brasileiras mostram um fraco desempenho desse setor, com a disponibilidade variando entre $52 \%$ e $66 \%{ }^{53,54}$. Diante desse cenário a associação entre seu consumo e a renda seria, até certo ponto, esperada.

Com relação aos indicadores da condição de saúde, os resultados revelam a existência de um gradiente, ou seja, a chance de utilizar medicamentos aumentou significativamente com o crescimento da necessidade, aqui medida pela autoavaliação do estado de saúde, pelo número de condições previamente diagnosticadas por médico e pela presença de problema de saúde nas duas últimas semanas. Estes resultados são consonantes com a literatura ${ }^{8-10,13}$. É importante salientar, por outro lado, que o uso concomitante de dois ou mais medicamentos deve ser monito- 
Tabela 5. Distribuição das razões de chance (OR) ajustadas e respectivos intervalos de confiança de 95\% (IC 95\%) obtidos na análise de regressão logística multinominal dos fatores associados à utilização de medicamentos. Hospital terciário, Rio de Janeiro, RJ, 2004.

\begin{tabular}{|c|c|c|}
\hline Variável & $\begin{array}{c}\text { Usou } 1 \\
\text { medicamento } \\
\text { OR (IC95\%) }\end{array}$ & $\begin{array}{c}\text { Usou } 20 u \text { mais } \\
\text { medicamentos } \\
\text { OR (IC95\%) }\end{array}$ \\
\hline \multicolumn{3}{|l|}{ Sexo } \\
\hline Masculino & 1,00 & 1,00 \\
\hline Feminino & $2,38(1,36-4,17)$ & $4,35(2,21-8,56)$ \\
\hline \multicolumn{3}{|c|}{ Renda pessoal mensal (salários mínimos) } \\
\hline$\leq 6$ & 1,00 & 1,00 \\
\hline Entre 6 e 12 & $2,08(1,09-3,97)$ & $1,28(0,59-2,78)$ \\
\hline$>12$ & $1,27(0,66-2,45)$ & $1,74(0,84-3,59)$ \\
\hline \multicolumn{3}{|l|}{ Autoavaliação de saúde } \\
\hline Muito boa/Boa & 1,00 & 1,00 \\
\hline Razoável & $1,52(0,85-2,72)$ & $2,37(1,15-4,87)$ \\
\hline Ruim/Muito ruim & $2,18(0,75-6,38)$ & $4,08(1,28-13,02)$ \\
\hline \multicolumn{3}{|c|}{ Número de condições diagnosticadas por médico } \\
\hline Nenhuma & 1,00 & 1,00 \\
\hline 1 & $1,51(0,82-2,78)$ & $2,33(1,08-5,05)$ \\
\hline $2 \mathrm{ou}+$ & $2,89(1,41-5,91)$ & $8,28(3,66-18,71)$ \\
\hline \multicolumn{3}{|c|}{ Apresentou problema de saúde nas duas últimas semanas } \\
\hline Não & 1,00 & 1,00 \\
\hline Sim & $2,10(1,05-4,19)$ & $3,13(1,51-6,52)$ \\
\hline
\end{tabular}

rado em função da potencialização do risco de ocorrência de reações adversas, de interações medicamentosas e de toxicidade ${ }^{55}$.

Neste grupo de trabalhadores o único fator específico, de cunho profissional do ambiente de trabalho, que esteve associado ao uso de medicamentos foi a licença por motivo de saúde relacionada ao trabalho nos últimos 12 meses, mas esta associação não se manteve no modelo ajustado. Boef-Cazou et al. ${ }^{11}$ na França, também não encontraram associação entre variáveis relacionadas ao trabalho e uso de medicamentos. Assim como na pesquisa francesa, também foi avaliado o uso global de medicamentos, o que pode ter contribuído para a não detecção de associação com as referidas variáveis. Estudo anterior encontrou associação entre a maior carga horária trabalhada na semana e o uso de anti-inflamatórios não esteroidais ${ }^{24}$, sugerindo a abordagem de classes específicas de medicamentos para ajudar a esclarecer determinadas associações.

A extrapolação dos resultados encontrados neste trabalho deve ser feita com cautela, já que estes se baseiam em uma população de funcionários de uma instituição de saúde. No entanto, é razoável imaginar que a parcela da população urbana e trabalhadora não deve diferir muito da estudada.

Para evitar vieses e garantir a validade interna do estudo, todos os procedimentos adotados para a coleta de dados foram padronizados (instrumentos, treinamento das equipes de campo, supervisão do trabalho de campo, entre outros). Estimulou-se intensamente a participação dos trabalhadores por meio de cartas, visitas aos setores de trabalho, mensagens em contracheques, website e cartazes.

É importante ressaltar ainda que a pesquisa envolvendo medicamentos pode ser prejudicada pela dificuldade inerente às dosagens e aos nomes dos produtos farmacêuticos, tanto químicos quanto comerciais. Nesta investigação duas estratégias foram adotadas para contornar esta dificuldade: (1) a solicitação de apresentação da embalagem e/ou receita para o(s) medicamento(s) consumido(s), o que também contribuiu para minimizar viés de memória e (2) a presença de estudantes de Farmácia para auxiliar no preenchimento desta parte específica do questionário.

Pelo nosso conhecimento, este é o primeiro estudo brasileiro dedicado a avaliar os determinantes do uso de medicamentos em população 
trabalhadora. Chama a atenção a alta prevalência de uso desses produtos nesta população, teoricamente mais saudável que a população em geral. Em particular, o maior uso de analgésicos requer atenção, pois muitos usuários costumam desconhecer ou relegar o fato de que tais fármacos, ainda que de venda livre, não são isentos de risco. As mulheres e os indivíduos em pior estado de saúde constituem os principais candidatos a programas de intervenção visando o uso adequado de produtos farmacêuticos.

\section{Colaboradores}

TCB Luz, VL Luiza, FG Avelar, YHM Hökerberg e SRL Passos participaram igualmente de todas as etapas de elaboração do artigo.

\section{Agradecimentos}

Ronaldo Fernandes e Marcio Danelon de Carvalho pelo apoio na classificação dos medicamentos e desenvolvimento dos bancos de dados.

\section{Referências}

1. VFA-Bio - Association of Research-Based Pharmaceutical Companies. The Pharmaceutical Market. [site na Internet]. [acessado 2010 out 15]. [Documento da Internet]. Disponível em: http://www. vfa.de/en/statistics/pharmaceuticalmarket/.

2. GRUPEMEF - Grupo dos Profissionais Executivos do Mercado Farmacêutico. Vendas Nominais em R\$, US\$ e em 1000 Unidades. [site na Internet]. [acessado 2010 out 15]. [Documento da Internet]. Disponível em: http://www.sindusfarmacomunica.org.br/uploads/ indicadores-economicos/aa 76bcc16bca $79 \mathrm{fc} 31 \mathrm{~b}$ 65 c5fd22e463f.xls

3. Barros JAC. Nuevas tendencias de la medicalización. Cien Saude Colet 2008;13(Supl.):579-587.

4. Aquino DS. Por que o uso racional de medicamentos deve ser uma prioridade? Cien Saude Colet 2008; 13(Supl.):733-736.

5. Lefréve F. A função simbólica dos medicamentos. Rev Saude Publica 1983;17(6):500-503.

6. Leite SN, Vieira M, Veber AP. Estudos de utilização de medicamentos: uma síntese de artigos publicados no Brasil e América Latina. Cien Saude Colet 2008; 13(Supl.):793-802.

7. Kongkaew C, Noyce PR, Ashcroft DM. Hospital admissions associated with adverse drug reactions: a systematic review of prospective observational studies. Ann Pharmacother. 2008; 42(7-8):1017-1025.

8. Loyola Filho AI, Uchoa E, Firmo JO, Lima-Costa MF. Estudo de base populacional sobre o consumo de medicamentos entre idosos: Projeto Bambui. Cad Saude Publica 2005; 21(2):545-553.

9. Carvalho MF, Pascom ARP, Souza-Júnior PRB, Damacena GN, Szwarcwald CL. Características da utilização de medicamentos na população brasileira, 2003. Cad Saude Publica 2005; 21(Supl.):S100S108.

10. Furu K, Straume B, Thelle DS. Legal drug use in a general population: Association with gender, morbidity, health care utilization, and lifestyle characteristics. J Clin Epidemiol. 1997; 50(3):341-349. 
11. Boeuf-Cazou O, Lapeyre-Mestre M, Niezborala M, Montastruc JL. Evolution of drug consumption in a sample of French workers since 1986: the 'Drugs and Work' study. Pharmacoepidemiol Drug Saf. 2009; 18(4):335-343.

12. Sihvo S, Klaukka T, Martikainen J, Hemminki E. Frequency of daily over-the-counter drug use and potential clinically significant over-the-counter-prescription drug interactions in the Finnish adult population. Eur J Clin Pharmacol. 2000; 56(6-7):495-499.

13. Arrais PSD, Brito LL, Barreto ML, Coelho HLL. Prevalência e fatores determinantes do consumo de medicamentos no Município de Fortaleza, Ceará, Brasil. Cad Saude Publica 2005; 21(6):1737-1746.

14. World Health Organization (WHO). How to Investigate the Use of Medicines by Consumers. Geneva: World Health Organization (WHO); 2004.

15. Holmgren K, Dahlin-Ivanoff S, Björkelund C, Hensing G. The prevalence of work-related stress, and its association with self-perceived health and sickleave, in a population of employed Swedish women. BMC Public Health. 2009; 9:73.

16. Cheng Y, Chen CW, Chen CJ, Chiang TL. Job insecurity and its association with health among employees in the Taiwanese general population. Soc Sci Med. 2005; 61(1):41-52.

17. Stansfeld SA, Fuhrer R, Shipley MJ, Marmot MG. Work characteristics predict psychiatric disorder: prospective results from the Whitehall II Study. Occup Environ Med. 1999; 56(5):302-307.

18. Lima Junior D. Avaliação das condições de trabalho em hospitais de Natal, Rio Grande do Norte, Brasil. Rev Esc Enf USP. 2009; 43(3):670-676.

19. Jinks AM, Lawson V, Daniels R. A survey of the health needs of hospital staff: implications for health care managers. J Nurs Manag. 2003; 11(5):343-350.

20. Kawano Y. Association of job-related stress factors with psychological and somatic symptoms among Japanese hospital nurses: effect of departmental environment in acute care hospitals. J Occup Health. 2008; 50(1):79-85.

21. Piko B. Burnout, role conflict, job satisfaction and psychosocial health among Hungarian health care staff: a questionnaire survey. Int J Nurs Stud 2006; 43(3):311-318.

22. Estryn-Behar M, Kaminski M, Peigne E, Bonnet N, Vaichere E, Gozlan C, Azoulay S, Giorgi M. Stress at work and mental health status among female hospital workers. Br J Ind Med. 1990; 47(1):20-28.

23. Sinokki M, Hinkka K, Ahola K, Koskinen S, Kivimäki M, Honkonen T, Puukka P, Klaukka T, Lönnqvist J, Virtanen M. The association of social support at work and in private life with mental health and antidepressant use: the Health 2000 Study. Affect Disord. 2009; 115(1-2):36-45.

24. Luz TCB, Rozenfeld S, Lopes CS, Faerstein E. Fatores associados ao uso de antiinflamatórios não esteróides em população de funcionários de uma universidade no Rio de Janeiro: Estudo Pró-Saúde. Rev. bras. epidemiol. 2006; 9(4):514-526.

25. Pelfrene E, Vlerickb P, Moreauc M, Mak RP, Kornitzerc M, Backer GD. Use of benzodiazepine drugs and perceived job stress in a cohort of working men and women in Belgium. Results from the BELSTRESS-study. Soc Sci Med. 2004; 59(2):433-442.
26. Acurcio FA, Rozenfeld S, Ribeiro AQ, Klein CH, Moura Cd, Andrade CR. Utilização de medicamentos por aposentados brasileiros. 1 - Metodologia e resultados de cobertura de inquérito multicêntrico. Cad Saude Publica 2006; 22(1):87-96.

27. Tomasi E, Sant'Anna GC, Oppelt AM, Petrini RM, Pereira IV, Sassi BT. Condições de trabalho e automedicação em profissionais da rede básica de saúde da zona urbana de Pelotas, RS. Rev. Bras. Epidemiol. 2007; 10(1):66-74.

28. Rozemberg B. O uso de calmantes e o "problema de nervos" entre lavradores. Rev Saude Publica 1994; 28(4):300-308.

29. Dicionário de especialidades farmacêuticas, DEF 20042005. 33a ed. Rio de Janeiro: Publicações Científicas; 2004

30. Agência Nacional de Vigilância Sanitária (ANVISA). Banco de dados de medicamentos da Agência Nacional de Vigilância Sanitária (ANVISA). [Site na Internet]. [acessado $2010 \mathrm{fev}$ ]. [Documento da Internet]. Disponível em: http://www.Anvisa.gov.br/ medicamentos/banco_med.htm.

31. World Health Organization (WHO). Collaborating Centre for Drug Statistics Methodology. Anatomical therapeutic chemical-ATC classification index with defined daily doses (DDDs). [acessado $2010 \mathrm{fev}$ ]. [Documento da Internet]. Disponível em: http:// www.whocc.no/atc_ddd_index.

32. Theme Filha MM, Szwarcwald CL, Souza Junior PR. Medidas de morbidade referida e inter-relações com dimensões de saúde. Rev Saude Publica 2008; 42(1):73-81.

33. Miilunpalo S, Vuori I, Oja P, Pasanen M, Urponen H. Self-rated health status as a health measure: the predictive value of self-reported health status on the use of physician services and on mortality in the working-age population. J Clin Epidemiol. 1997; 50(5):517-528.

34. Moller L, Kristensen TS, Hollnagel H. Self rated health as a predictor of coronary heart disease in Copenhagen, Denmark. J Epidemiol Community Health 1996; 50(4):423-428.

35. Brasil. Ministerio do Trabalho e Emprego (MTE). Classificação Brasileira de Ocupações (CBO 2002). [Site na Internet]. [acessado 2009 jun]. Disponível em http://www.mtecbo.gov.br

36. Long JS, Freese J. Regression models for categorical dependent variables using Stata. College Station: Stata Press; 2003.

37. Carrasco-Garrido P, Jiménez-García R, Barrera VH, Miguel AGd. Predictive factors of self-medicated drug use among the Spanish adult population. Pharmacoepidemiol Drug Saf 2008; 17(2):193-199.

38. Loyola Filho AI, Uchoa E, Lima-Costa MF. Estudo epidemiologico de base populacional sobre uso de medicamentos entre idosos na Regiao Metropolitana de Belo Horizonte, Minas Gerais, Brasil. Cad Saude Publica 2006; 22(12):2657-2667.

39. Rothman KJ, Greenland S, Lash TL. Modern Epidemiology. $3^{\text {rd }}$ ed. Philadelphia: Lipincott Williams \& Wilkins; 2008.

40. De Lucia R, Planeta CS, Almeida NS. Consumo de medicamentos, bebidas alcoólicas e cigarros por operários de Cubatäo. AMB Rev Assoc Med Bras. 1987; 33(11-12):215-218. 
41. De Lucia R, Planeta CS, Simöes NA, Medrado Faria MA. Trabalho em turno: consumo de medicamentos, bebidas alcoólicas e tabaco por operários de Cubatäo - SP. Rev. bras. saúde ocup. 1988; 16(64):5153.

42. World Health Organization (WHO). Introduction to Drug Utilization Research. Geneva: World Health Organization (WHO); 2003.

43. Katsarava Z, Diener HC, Limmroth V. Medication overuse headache: a focus on analgesics, ergot alkaloids and triptans. Drug Saf 2001; 24(12):921-927.

44. Wolff T, Miller T, Ko S. Aspirin for the primary prevention of cardiovascular events: an update of the evidence for the U.S. Preventive Services Task Force. Ann Intern Med. 2009; 150(6):405-410.

45. Tolman KG. Hepatotoxicity of non-narcotic analgesics. Am J Med. 1998; 105(1B):13S-19S.

46. Perneger TV, Whelton PK, Klag MJ. Risk of kidney failure associated with the use of acetaminophen, aspirin, and nonsteroidal antiinflammatory drugs. New Engl J Med. 1994; 331(25):1675-1679.

47. Sans S, Paluzie G, Puig T, Balaña L, Balaguer-Vintró I. Prevalencia del consumo de medicamentos en la población adulta de Cataluña. Gac Sanit. 2002; 16(2):121-130.

48. Laukkanen P, Heikkinen E, Kauppinen M, Kallinen M. Use of drugs by non-institutionalized urban Finns born in 1904-1923 and the association of drug use with mood and self-rated health. Age Ageing. 1992; 21(5):343-352.

49. Lima MG, Ribeiro AQ, Acurcio FA, Rozenfeld S, Klein $\mathrm{CH}$. Composição dos gastos privados com medicamentos utilizados por aposentados e pensionistas com idade igual ou superior a 60 anos em Belo Horizonte, Minas Gerais, Brasil. Cad Saude Publica 2007; 23(6):1423-1430.

50. Lundberg L, Johannesson M, Isacson DG, Borgquist L. Effects of user charges on the use of prescription medicines in different socio-economic groups. Health Policy 1998; 44(2):123-134.

51. Dewa CS, Hoch JS, Steele L. Prescription drug benefits and Canada's uninsured. Int J Law Psychiatry. 2005; 28(5):496-513.

52. Silveira FG, Osório RG, Piola SF. Os gastos das famílias com saúde. Cien Saude Colet 2002; 7(4):719-731.

53. Guerra Jr AA, Acúrcio FA, Gomes CAP, Miralles M, Girardi SN, Werneck GAF, Carvalho CL. Disponibilidade de medicamentos essenciais em duas regiões de Minas Gerais, Brasil. Rev Panam Salud Publica 2004; 15(3):168-175.

54. Organização Panamericana de Saúde (OPAS). Avaliação da Assistência Farmacêutica no Brasil: Estrutura, Processos e Resultados. Brasília: Organização Panamericana de Saúde (OPAS); 2005.

55. Osorio-de-Castro CGS, Castilho SR, Peixoto MAP, Mosegui GBG, Luiza VL. Estudos de Utilização de Medicamentos: Noções Básicas. $1^{\text {a }}$ ed. Rio de Janeiro: Editora Fiocruz; 2000.

Artigo apresentado em 15/10/2010

Aprovado em 19/03/2011

Versão final apresentada em 06/04/2011 